\title{
Antineutrino and gamma emission from the OSIRIS research reactor
}

\author{
Lydie Giot ${ }^{\mathrm{a}}$ and Muriel Fallot \\ SUBATECH, CNRS/IN2P3, Univ. de Nantes, EMN, 44307 Nantes, France
}

\begin{abstract}
For the first time, the summation method has been coupled with a complete reactor model, in order to predict the antineutrino emission of a research reactor. This work, discussed in the first part of this paper, allows us to predict the low energy part of the antineutrino spectrum, evidencing the important contribution of actinides to the antineutrino emission. Experimental conditions at short distance from research reactors are challenging, because the reactor itself produces huge gamma background that induce accidental and correlated backgrounds in an antineutrino target. The understanding of this background is of utmost importance and triggered the second part of the work presented here.
\end{abstract}

\section{Introduction}

Antineutrinos arise from the fission product beta decays and are emitted in huge quantities by reactor cores: around $10^{19}$ antineutrinos/sec for a $100 \mathrm{MWe}$ research reactor. Their energy spectrum and flux reflect the composition of the fuel, but also its thermal power [1,2]. The International Atomic Energy Agency (IAEA) has expressed its interest in the potentialities of antineutrino detection as a new tool for reactor monitoring. Worldwide effort has led to further investigations into the feasibility of using such detectors for safeguards purposes. The reactor antineutrinos have also been used by many particle physicists since the late fifties to study the neutrino properties. With the advance of next generation of reactor neutrino experiments, new calculations of the main uranium and plutonium isotope antineutrino energy spectra were performed. The conversion procedure to obtain antineutrino energy spectra was revisited and led to the so-called "reactor anomaly" [3,4]. Several hypotheses are raised to explain this anomaly, among which the existence of light sterile neutrinos [5].

Since then, experimental projects have been born in the US, Canada, Russia, Great Britain and France, aiming at installing an antineutrino detector close to a research reactor in order to evidence the existence of such sterile neutrinos and/or safeguards purposes [6]. Most of research reactors use fuel enriched to about $20 \%$ in ${ }^{235} \mathrm{U}$, which is a limit imposed by the International Atomic Energy Agency. This enrichment guarantees that most of the fissions arises from ${ }^{235} \mathrm{U}$ thermal fission, but there may be a small contribution of fissions arising from ${ }^{239} \mathrm{Pu}$ produced during irradiation from the huge amount of ${ }^{238} \mathrm{U}$ present in the fuel. This contribution has to be quantified as the ${ }^{239} \mathrm{Pu}$ antineutrino energy spectrum shape differs from the ${ }^{235} \mathrm{U}$ one. Sterile neutrino searches at research reactors will first use an analysis in shape of the measured antineutrino energy spectra as a function of the distance

a e-mail: lydie.giot@subatech.in2p3.fr from the reactor. An accurate prediction of the antineutrino energy spectrum emitted by the research reactor will be needed. In the case of the NUCIFER experiment installed closed to the research OSIRIS reactor in France [7], the antineutrino energy spectrum and flux were computed for the first time using the summation method, coupled to a depletion calculation of the reactor core.

Moreover, the experimental conditions at short distance from research reactors are difficult to handle. Huge quantities of gamma are induced and at the origin of accidental/correlated backgrounds in the antineutrino target. The gamma energy spectra produced by the OSIRIS reactor was estimated using the developed reactor simulation and after then propagated up to the antineutrino detector location to estimate the gamma flux rate.

First, the simulation of the OSIRIS reactor done with the MURE code will be introduced. Secondly, the calculation of the antineutrino spectrum using the summation method will be detailed. Then part of the work performed on the gamma background of the OSIRIS reactor will be presented.

\section{The MURE simulation of the OSIRIS reactor and the associated MURE simulation}

\subsection{The OSIRIS research reactor}

The OSIRIS reactor (CEA-Saclay, France) is a light water $70 \mathrm{MW}$ reactor with an open core $\left(57 \times 57 \times 60 \mathrm{~cm}^{3}\right)$. The core vessel is surrounded by a pool of $11 \mathrm{~m}$ deep, $7.5 \mathrm{~m}$ long and $6.5 \mathrm{~m}$ wide. Its geometry is displayed in Fig. 1. The core contains a rack of 56 cells, loaded with 38 standard fuel elements with plates, 6 control command rods, 5 locations for irradiation experiments named Chouca ovens. An internal wall (7 cells) and an external wall to the vessel of $5 \mathrm{~cm}$ thick in Beryllium are located on the southern face of the core and act as reflectors. The fuel elements are made with 22 standard fuel plates in a $\mathrm{U}_{3} \mathrm{Si}_{2} \mathrm{Al}$ alloy, enriched in ${ }^{235} \mathrm{U}$ to $19.75 \%$.

(C) The Authors, published by EDP Sciences. This is an Open Access article distributed under the terms of the Creative Commons Attribution License 4.0 (http://creativecommons.org/licenses/by/4.0/). 


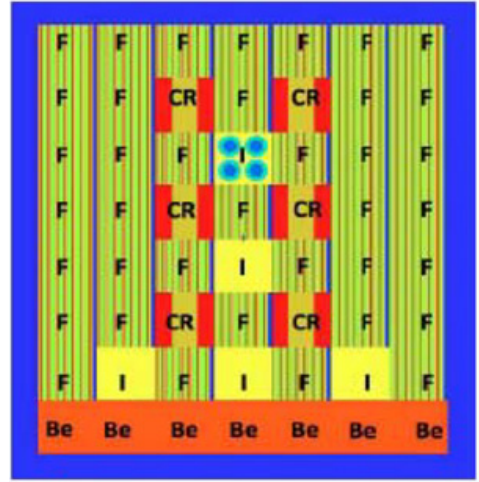

Figure 1. Osiris core reactor simulated with the MURE code. The colours represent the different materials and universes defined in the core model. One can see the structure of the fuel elements $(\mathrm{F})$, the Beryllium walls (Be), the control rods (CR) and the irradiaton locations (I) with one filled in with 4 Chouca ovens.

The upper part of the control command rods is made in Hafnium, an absorbant material. The reactor delivers a neutron flux of $10^{18}$ neutrons. $\mathrm{cm}^{2} . \mathrm{s}^{-1}$ for about 200 days per year as an average, with cycles of 3 to 5 weeks. The NUCIFER detector is placed at $7 \mathrm{~m}$ from the core behind a concrete wall of $2 \mathrm{~m}$ [7].

\subsection{The associated MURE reactor simulation}

The reactor simulation was performed with the Monte Carlo Code MURE (MCNP Utility for Reactor Evolution). Available at the NEA databank, the MURE code is a precision, open-source code, designed by CNRS/IN2P3 laboratories and written in $\mathrm{C}++$ which automates the preparation, computation of successive MCNP (Monte Carlo N-Particle) calculations and solves the Bateman equations in between, using a Runge-Kutta method, for burnup purpose [8]. The MURE code is highly flexible to simulate reactors with a variety of refueling schemes, operation history and non-trivial core geometry like research reactors. The SUBATECH laboratory has developed important new functions in MURE to analyze the beta decay properties of the fission products in order to compute the associated antineutrino energy spectrum together with the reactor fuel evolution. Especially, a detailed full core model with MURE following the operation history of the reactor plus the estimate of the associated systematic errors was developed for the Double Chooz experiment [9].

In the reactor model presented here, all the fuel elements were treated as evolving materials. The core was divided in seven evolving materials, called universes in order to take into account its refueling by 1 seventh every 20 days, for a simulated period of 280 days. The input nuclear cross sections were taken from the ENDFB/VI library in first priority and a thermal $\mathrm{S}(\alpha, \beta)$ treatment was applied. This simulation was benchmarked with APOLLO2 calculations of the neutron flux in one of the irradiation devices for a fresh fuel core [10]. The overall shape of the neutron energy spectrum is well reproduced by the MURE calculation. Differences in the results normalisation arise from the unknown operation history associated to the APOLLO2 simulation and database choices. However, the ratio betwen the neutron fluxes in

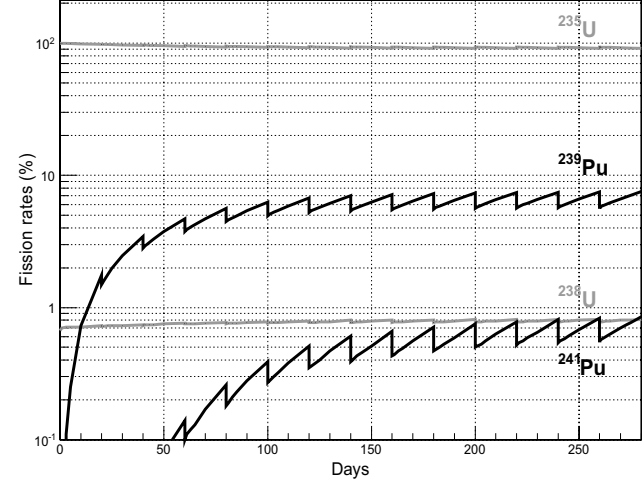

Figure 2. Fission rates distribution for the 4 main fissile nuclei: ${ }^{235} \mathrm{U},{ }^{238} \mathrm{U},{ }^{239} \mathrm{Pu}$ and ${ }^{241} \mathrm{Pu}$ with a refueling scenario of 20 days.

the thermal and rapid energy ranges are in good agreement, as shown in Ref. [11].

The distribution of the fission rates for the 4 main fissile nuclei: ${ }^{235} \mathrm{U},{ }^{238} \mathrm{U},{ }^{239} \mathrm{Pu}$ and ${ }^{241} \mathrm{Pu}$ is presented in Fig. 2 for a refueling scenario by 1 seventh every 20 days, starting with a core completly filled with fresh fuel. The fission rates are constrained by the power maintained at its nominal value along the evolution. By definition, the equilibrium of the reactor is reached when the fission rates show a periodic pattern repeated every 20 days. This is reached for the different contributions after 260 days, i.e., the beginning of the third refueling cycle for the different fuel blocks. For this reactor with a fuel composition at equilibrium, the main contributor to the total amount of fissions in-core is ${ }^{235} \mathrm{U}$. For this isotope, the variation of the fission rate between the beginning and the end of the cycle is about $2-3 \%$.

\section{Antineutrino spectrum}

\subsection{The summation method}

The antineutrino energy spectrum and flux are computed with the summation method [12]. The number of emitted antineutrinos produced in a reactor core over the time $t$ is defined by :

$$
N_{\bar{\nu}}(E)=\sum_{n} Y_{n}(Z, A, t) . \sum_{i} S_{\bar{v}, n, i}(Z, A, E)
$$

where $Y_{n}(Z, A, t)$ : the activity of a beta minus emitter is weighted by the associated antineutrino spectrum. The antineutrino spectrum of one beta emitter is the sum over the different branches of all $\beta^{-} / \bar{\nu}$ decay spectra. The MURE simulation of the OSIRIS reactor is used to provide the amount in-core of all beta minus emitters as a function of time, allowing for the first time to take into account all the contributions, i.e., not only the fission products but also the contribution of the actinides and heavy nuclei produced during core operation. The nuclear databases are the same as the ones presented in [12], including the latest published Total Absorption Spectroscopy experimental results.

\subsection{Results}

The antineutrino emitted flux computed with the summation method described above is presented on Fig. 3 (plain lines). The colors correspond to a fuel evolution of 201, 


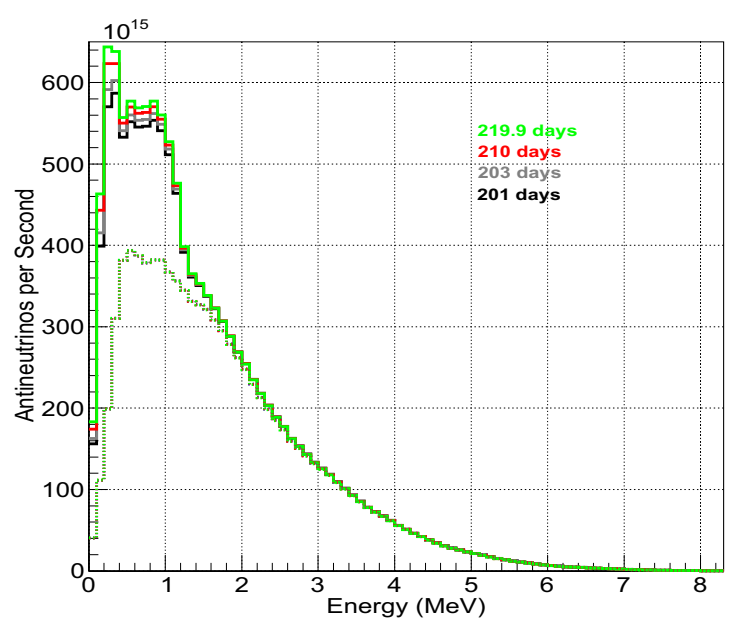

Figure 3. Emitted antineutrino energy spectra computed with the complete core model (plain lines) and with individual summation method spectra for ${ }^{235} \mathrm{U},{ }^{239} \mathrm{Pu},{ }^{238} \mathrm{U}$ and ${ }^{241} \mathrm{Pu}$ weighted by the fission rates computed with the same core simulation (dashed lines) for the following time after core start-up: 201 days, 203, 210 and 219.9 days. Results for dashed lines are superposed.

203, 210 and 219.9 days with a periodic refueling by seventh every 20 days.

A second set of calculations, also presented on Fig. 3 with dashed lines, was performed with a method similar to the one classicly used by reactor antineutrino experiments. The individual antineutrino spectra from the main uranium and plutonium isotopes are weighted by their fission rates in the following way:

$$
N_{\bar{v}}(E)=\frac{P(t)}{\Sigma_{k} \alpha_{k}(t) \cdot E_{k}} \cdot \sum_{k} \alpha_{k}(t) \cdot S_{k}(E)
$$

where the first term accounting for the number of fissions occuring over the time $t$ is the ratio of the thermal power over the average energy released per fission of the main four fissile isotopes present in the fuel: ${ }^{235} \mathrm{U},{ }^{238} \mathrm{U},{ }^{239} \mathrm{Pu}$ and ${ }^{241} \mathrm{Pu} . \alpha_{k}(t)$ stands for the percentage of fissions undergone by the isotope $\mathrm{k}$ and is given by the MURE reactor calculation. The mean energy released per fission, $E_{k}$ is taken from Kopeikin et al. [13]. The individual spectra $S_{k}(E)$ are computed with the summation method to match the irradiation time (12 hours for ${ }^{235} \mathrm{U}$ and 36 hours for the three other fissible nuclei) of the individual beta energy spectra of the main uranium and plutonium isotopes measured at ILL [14-17] and Garching [18].

The emitted antineutrino flux, presented on Fig. 3, computed with the complete core model associated to the summation method is much larger than the one using the individual energy spectra of ${ }^{235} \mathrm{U},{ }^{238} \mathrm{U},{ }^{239} \mathrm{Pu}$ and ${ }^{241} \mathrm{Pu}$ computed with the summation method. This is due to the contribution of ${ }^{239} \mathrm{U}$ and ${ }^{239} \mathrm{~Np}$ beta decays at low energy.

One can notice that regarding the spectra computed using the individual uranium and plutonium spectra, the time evolution has no impact; the four dashed line spectra are superposed. This is expected as these individual spectra have been computed without off equilibrium effects or other reactor physics effects, and after a fixed irradiation time. The discrepancy between both calculations in terms of normalization and evolution with time is clearly evidenced, due to the following effects: contribution of actinides that can be accounted for only in the first method, and off-equilibrium effects which include the effect of neutron capture on the fission product concentrations and the time evolution of their concentrations in-core. Antineutrino experiments installed at research reactors using Low Enriched Uranium fuel will have to cope with such effects.

\section{Gamma flux}

In a reactor environment, the gamma sources are numerous: prompt gamma rays emitted when the fission process occurs, $\gamma$ decays of the fission fragments after their $\beta^{-}$decays, $(\mathrm{n}, \gamma)$ reactions in the fuel or in different reactor components, and more generally gamma rays emitted by the excited nuclei created in the different processes. In order to understand in details the gamma background of the NUCIFER experiment, a simulation of the OSIRIS reactor surrounded by its pool and the concrete walls up to the casemate where the antineutrino detector is located, was performed. To transport gammas with enough statistics to the casemate, the pool and concrete walls were discretized in several layers of $50 \mathrm{~cm}$ and a variance reduction technique used.

The chosen strategy was to perform simulations with only the reactor core $(\mathrm{C})$, core and pool $(\mathrm{CP})$, corepool plus concrete walls (CPW), separately with the MCNPX2.7.d code using preferentially the ENDFB/VI cross-section library and the ENDFB/VII one for photonuclear reactions. As a first step, in each of the three cases $(\mathrm{C}, \mathrm{CP}, \mathrm{CPW})$, a reference simulation using the default physics options of MCNPX 2.7.d for the gamma transport was done in order to carry out the simplest simulation with the following reactions: Compton scattering, pair production, Thomson and Rayleigh scatterings, photoelectric effect plus $\mathrm{K}$ and $\mathrm{L}$ shell flurorescences. In a second step to estimate their respective contributions in the gamma production process, extra simulations were performed by adding only one additional physics phenomenon at a time: delayed gamma rays, Bremsstrahlung, photonuclear reactions. For instance, this work allowed us to understand the importance of the photonuclear reactions which occur in the concrete walls, mostly $(\gamma, n)$ reactions on ${ }^{40} K$. This secondary neutron production could partly explain the neutron background measured in the detector casemate by the Nucifer collaboration and which increases with the reactor power.

In Fig. 4, the gamma flux rate expected in the detector casemate is calculated for a reactor cycle of 20 days considering a fuel composition at equilibrium obtained with the refueling scenario presented in the section II. No energy threshold is applied on the gamma detection. The error bars are statistical and computed using the number of $\gamma$ tracks. From this simulation, the gamma flux rate in an antineutrinos detector of $10 \mathrm{~m}^{2}$ surface is estimated to be 2.5 MHz. A more realistic fuel composition could be used in the future to improve the normalisation of the gamma flux rate but this first result is already in good agreement with the order of magnitude of $5 \mathrm{MHz}$ measured in the casemate by the NUCIFER collaboration. 


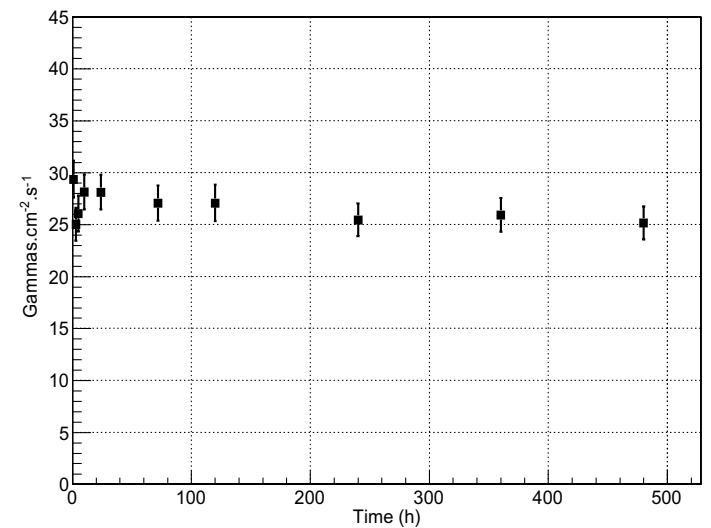

Figure 4. Gamma flux rate in the detector casemate as a function of time, computed with MURE from a reactor fuel composition at the equilibrium.

\section{Conclusion}

The simulation of the OSIRIS research reactor was performed with the MURE code, including the core, the pool and an antineutrino detector casemate. The implementation of the periodic refueling of the core every 20 days by seventh made it possible to carry out a first study of the emitted antineutrino spectrum which could be measured at such a research reactor. The antineutrino energy spectrum and flux were computed using the summation method, coupled for the first time to a reactor core in evolution. The emitted antineutrino energy spectrum associated to the reactor fuel at equilibrium is provided and could be used by experiments studying fundamental properties of neutrinos at such facilities. Off-equilibrium effects affecting the antineutrino spectral shape due to neutron capture and time evolution of the fission product concentrations in-core were computed, as well as the impact of actinides created along the operation of the reactor. In a second part, a detailed study of the gamma ray background was performed. Overall the model succeeded to reproduce the gamma background rate measured at the location of the Nucifer detector. These results could be used to compute the sensitivity of short baseline reactor antineutrino experiments foreseen in the frame of the search of potential sterile neutrinos.

The authors thank the NEEDS challenge and the GEDEPEON research groupment for their financial support and the people from the OSIRIS reactor for providing useful information.

\section{References}

[1] L.A. Mikaelian, Proc. Int. Conf. Neutrino-77 2, 383 (1978)

[2] V.A. Korovkin et al., At. Energy 65, 712 (1988)

[3] Th. A. Mueller et al., Phys. Rev. C 83, 054615 (2011)

[4] G. Mention et al., Phys. Rev. D 83, 073006 (2011)

[5] K.N. Abazajian et al., "Light Sterile neutrino: a white paper", arXiv: 1204.5379

[6] M. Fallot, Nuclear Data Sheets 120, 13 (2014)

[7] G. Boireau et al., Phys. Rev. D 93, 112006 (2016)

[8] O. Meplan. Tech. Rep. LPSC 0912 and IPNO09-01 (2009), http://www.nea.fr/tools/ abstract/detail/nea-1845

[9] A. Onillon, PhD Thesis, Ecole des Mines de Nantes (2014). Article in preparation

[10] F. Malouch, Thèse de Doctorat, Rapport CEA R6034, 2003

[11] V.M. Bui, L. Giot, M. Fallot et al., arXiv: 1602.07522

[12] M. Fallot et al., Phys. Rev. Lett 109, 202504 (2012)

[13] V. Kopeikin et al., Phys. At. Nucl. 67, 1892 (2004)

[14] K. Schreckenbach et al., Phys. Lett. 99B, 251 (1981)

[15] K. Schreckenbach et al., Phys. Lett. 160B, 325 (1985)

[16] F. von Feilitzsch, A. A. Hahn and K. Schreckenbach, Phys. Lett. 118B, 162 (1982)

[17] A.A. Hahn et al., Phys. Lett. B218, 365 (1989)

[18] N. Haag et al., Phys. Rev. Lett. 112, 122501 (2014) 